\title{
A PROBABILISTIC FRAMEWORK FOR AUTOMATIC PROSTATE SEGMENTATION WITH A STATISTICAL MODEL OF SHAPE AND APPEARANCE
}

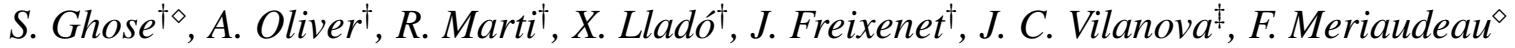 \\ $\dagger$ University of Girona, Computer Vision and Robotics Group, Girona, Spain. \\ ¥ Girona Magnetic Resonance Center, Girona, Spain. \\ $\diamond$ Université de Bourgogne, Le2i-UMR CNRS 5158, Le Creusot, France. \\ sghose@eia.udg.edu,soumyaghose@gmail.com
}

\begin{abstract}
Prostate volume estimation from segmented prostate contours in Trans Rectal Ultrasound (TRUS) images aids in diagnosis and treatment of prostate diseases, including prostate cancer. However, accurate, computationally efficient and automatic segmentation of the prostate in TRUS images is a challenging task owing to low Signal-To-Noise-Ratio (SNR), speckle noise, micro-calcifications and heterogeneous intensity distribution inside the prostate region. In this paper, we propose a probabilistic framework for propagation of a parametric model derived from Principal Component Analysis (PCA) of prior shape and posterior probability values to achieve the prostate segmentation. The proposed method achieves a mean Dice similarity coefficient value of $0.96 \pm 0.01$, and a mean absolute distance value of $0.80 \pm 0.24 \mathrm{~mm}$ when validated with 24 images from 6 datasets in a leave-one-patient-out validation framework. Our proposed model is automatic, and performs accurate prostate segmentation in presence of intensity heterogeneity and imaging artifacts.
\end{abstract}

Index Terms - Prostate Segmentation, Expectation Maximization, Bayes Classification, Active Appearance Model.

\section{INTRODUCTION}

Prostate cancer affects life of over 670,000 people worldwide, accounting for over 32,000 deaths in North America [1]. Prostate volume determined from segmented TRUS images serves as an important parameter in determining presence of benign or malignant tumor during diagnosis of prostate diseases [2]. However, manual segmentation of the prostate from TRUS images is time consuming and suffers from inter and intra observer variabilities and personal biases. Computer aided semi-automatic or automatic segmentation of the prostate from TRUS images is also a challenging task, due to low SNR, speckle noise, imaging artifacts and heterogeneous intensity distribution inside the prostate region.

Thanks to VALTEC 08-1-0039 of Generalitat de Catalunya, Spain and Conseil Régional de Bourgogne, France for funding.
To address the challenges involved with prostate segmentation in 2D TRUS images, we propose a novel Active Appearance Model (AAM) [3] that is trained, initialized and propagated by the probabilistic value of a pixel being prostate given its position and intensity obtained in a Bayesian framework [4]. The performance of our method is validated using 24 images from 6 datasets in a leave-one-patient-out validation framework, and it is compared to traditional AAM [3], our previous work on texture guided AAM [5] and with some of the works of the state-of-the-art. Experimental results show that our method performs accurate TRUS prostate segmentation, obtaining better performances compared to [3], and [5] and comparable to some works in literature $[6,7,8,9]$. The key contributions of this work are:

- Use of the likelihood information obtained from prostate gland pixel intensities and positions in a Bayesian framework to obtain a probabilistic representation of the prostate region.

- Use of the probabilistic information in training, automatic initialization and propagation of a statistical model of shape and appearance that improves on computational time and segmentation accuracy when compared to the traditional active appearance model [3].

To the best of our knowledge this is the first attempt to use probabilistic information of the prostate region from a Bayesian framework in training and propagation of AAM. The rest of the paper is organized as follows. The probabilistic framework for automatic initialization and propagation of AAM is formulated in Section 2 followed by the presentation of quantitative and qualitative evaluation of our method in Section 3. We finally draw conclusions in Section 4.

\section{OUR PROPOSED METHOD}

The proposed method is developed on three major components: using an Expectation Maximization (EM) model to determine prior class (prostate or background) probability from 


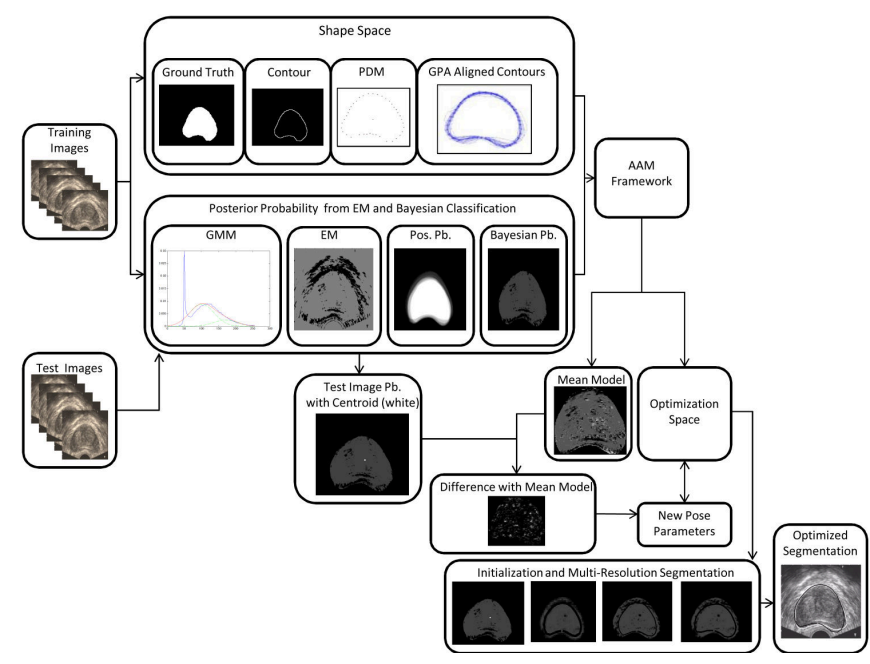

Fig. 1. Schematic representation of our approach. Abbreviations used PDM $=$ Point Distribution Model, GPA = Generalized Procustes Analysis, GMM $=$ Gaussian Mixture Model, EM $=$ Expectation Maximization, Pos. $=$ Positional, $\mathrm{Pb} .=$ Probability.

pixel intensities, a Bayesian framework for obtaining posterior probability distribution from prior probabilities of the EM and the pixel position and finally, the adaptation of traditional AAM for incorporating probabilistic values for training, initialization and propagation. The schema of our proposed method is illustrated in Fig. 1.

\subsection{Expectation Maximization and Prior Probabilities}

The probability of a pixel intensity being prostate is obtained in an EM [4] framework. Given a model $X$ of observed data, a set of latent unobserved data $Z$ and a vector of unknown parameters $\theta$, along with a likelihood function $L(\theta ; X, Z)$, the EM algorithm seeks to find the maximum likelihood estimate by iteratively applying the expectation and the maximization steps. In Eq. (1), the expectation step calculates the expected value of the log likelihood function with current estimated parameters $\theta^{t}$ and in Eq. (2), the maximization step find the parameters that maximizes this quantity.

$$
\begin{aligned}
Q\left(\theta \mid \theta^{t}\right) & =E_{Z \mid X, \theta^{t}}[\log L(\theta ; X, Z)] \\
\theta^{t+1} & =\operatorname{argmax}_{\theta}\left[Q\left(\theta \mid \theta^{t}\right)\right]
\end{aligned}
$$

In our model the intensity histogram of a TRUS image (Fig. 2(a)) is approximated with two class (prostate and background) Gaussian mixture model (Fig. 2(b)). Maximum a posteriori estimates of the class means and standard deviations are used to soft cluster the pixels, assigning probabilistic membership values of being in either classes (Fig. 2(c)). The likelihood of a pixel location in an image being prostate (Fig. $2(d)$ ) is obtained by normalizing the ground truth values of all the pixels for all the training images as,

$$
P\left(x_{p s} \mid C_{p r s}\right)=\frac{1}{N} \sum_{i=1}^{N} G T_{i}
$$

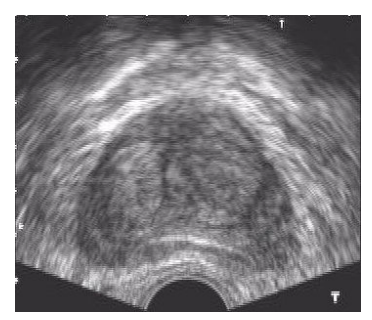

(a) (b)

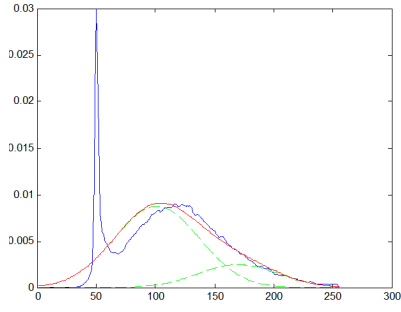

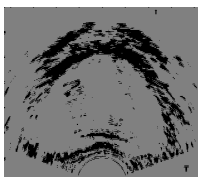

(c)

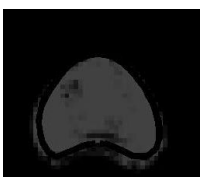

(g)

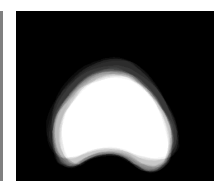

(d)

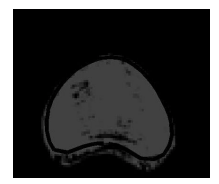

(h)

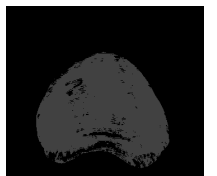

(e)

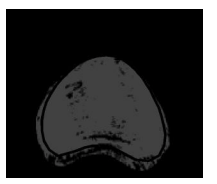

(i)

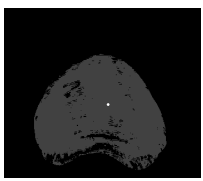

(f)

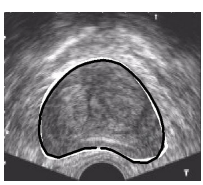

(j)
Fig. 2. Bayesian framework for segmentation (b) Intensity histogram (blue line), Gaussian mixture model (red line), and two Gaussian class (green line) (c) Output of EM of image (a). (d) Prior probability of pixels position in an image being prostate, (e) Posterior probability of a pixel being prostate after Bayes classification, (f) Centroid (white dot) computed from probability values for AAM initialization. On initialization the AAM segments prostate in a multi-resolution framework 2(g), 2(h) and 2(i) to give final segmentation 2(j).

where $G T$ represents ground truth of training images. In our model the class prior probability is estimated from the frequency of the pixels belonging to a class as,

$$
P\left(C_{\text {Prostate }}\right)=\frac{\sum_{i=1}^{n} x_{i}}{\sum_{j=1}^{m} x_{j}}
$$

where $x_{i}$ represents all the pixels belonging to prostate region and $x_{j}$ represents all the pixels in all training images.

\subsection{The Bayesian Framework}

The prior probabilities of intensity, location and class prior probabilities are used in a Bayesian framework to achieve a Bayesian classification of the pixels (Fig. 2(e)). We consider a pixel in TRUS image to be a $n$-dimensional feature vector $X=\left(x_{1}, x_{2}, x_{3}, \ldots . ., x_{n}\right)$. According to the Bayes rule,

$$
P\left(C_{i} \mid X\right)=\frac{P\left(X \mid C_{i}\right) P\left(C_{i}\right)}{P(X)}
$$

where $P\left(C_{i} \mid X\right)$ represents posterior probability distribution of a class given the prior $P\left(C_{i}\right)$ (i.e. $P\left(C_{\text {Prostate }}\right)$ ) and the likelihood $P\left(X \mid C_{i}\right)$. $P(X)$ being equal for all classes could be removed from the formulation. Considering class conditional independence the likelihood could be formalized as,

$$
\begin{aligned}
P\left(X \mid C_{i}\right) & =\prod_{K=1}^{n} P\left(x_{k} \mid C_{i}\right) \\
& =P\left(x_{p s} \mid C_{p r s}\right) \cdot P\left(x_{i n} \mid C_{p r s}\right)
\end{aligned}
$$


Table 1. Quantitative Comparison of AAMs

\begin{tabular}{|l|l|l|l|l|l|l|l|}
\hline Method & DSC & HD(mm) & MAD $(\mathrm{mm})$ & MaxD $(\mathrm{mm})$ & Specificity & Sensitivity & Accuracy \\
\hline AAM [3] & $0.94 \pm 0.03$ & $4.92 \pm 0.96$ & $2.15 \pm 0.94$ & $5.3 \pm 0.48$ & $0.89 \pm 0.03$ & $0.993 \pm 0.006$ & $0.97 \pm 0.009$ \\
\hline Ghose et al. [5] & $0.95 \pm 0.01$ & $5.08 \pm 1.18$ & $1.48 \pm 0.36$ & $5.01 \pm 1.13$ & $0.92 \pm 0.02$ & $0.998 \pm 0.001$ & $0.97 \pm 0.01$ \\
\hline Our Method & $0.96 \pm 0.01$ & $2.80 \pm 0.86$ & $0.80 \pm 0.24$ & $2.79 \pm 0.80$ & $0.94 \pm 0.01$ & $0.991 \pm 0.004$ & $0.98 \pm 0.005$ \\
\hline
\end{tabular}

where the likelihood $P\left(X \mid C_{i}\right)$ is obtained from the product of the probability of a pixel intensity being prostate $\left(P\left(x_{i n} \mid C_{p r s}\right)\right)$ from EM framework and the probability of a pixel location being prostate $\left(P\left(x_{p s} \mid C_{p r s}\right)\right)$ i.e. obtained by normalizing the ground truth values.

\subsection{Bayesian Guided Active Appearance Model}

AAM provides a compact framework built from a priori shape and texture variabilities knowledge acquired from training images to segment an unseen image exploiting the prior knowledge of the optimization space. The process of building AAM could be subdivided into three major components: building the shape model, building the texture model and building the combined shape and texture model.

The Point Distribution Model (PDM) [3] is built from manually segmented contours, which are aligned to a common reference frame with Generalized Procrustes Analysis (GPA). Principal Component Analysis (PCA) of the aligned PDMs identifies the principal modes of shape variations. Intensity distribution are warped into correspondence using a piece wise affine warp and sampled from shape free reference. PCA of the intensity distribution is used to identify the principal modes of intensity variations. The model may be formalized in the following manner: In Eq. (7) let $E\{s\}$ and $E\{t\}$ represent the shape and intensity models,

$$
\begin{array}{r}
E\{s\}=\bar{s}+\Phi_{s} \theta \\
E\{t\}=\bar{t}+\Phi_{t} \theta
\end{array}
$$

where $s$ and $t$ are the shape and the intensities of the corresponding training images, $\bar{s}$ and $\bar{t}$ denote the mean shape and intensity respectively, then $\Phi_{s}$ and $\Phi_{t}$ contain the first $p$ eigenvectors of the estimated joint dispersion matrix of shape and intensity and $\theta$ represents the corresponding eigenvalues. The model of shape and intensity variations are combined in a linear framework and a third PCA ensures the reduction in redundancy of the combined model. In addition to the parameters $\theta$, four parameters, two translations, rotation and scale are represented by $\psi$. In order to infer the parameter values of $\theta$ and $\psi$ of a previously unseen image, a Gaussian error model between model and pixel intensities is assumed. Furthermore, a linear relationship between changes in parameters and difference between model and image pixel intensities $\Delta t$ is assumed as,

$$
\Delta t=X\left[\begin{array}{c}
\Delta \psi \\
\Delta \theta
\end{array}\right]
$$

$X$ is estimated from weighted averaging over perturbation of model parameters and training examples. Eq. 8 is solved in least square manner fitting error as,

$$
\left[\begin{array}{c}
\widehat{\psi} \\
\widehat{\theta}
\end{array}\right]=\left(X^{T} X\right)^{-1} X^{T} \delta t
$$

We propose to use the probability values obtained from Bayes classification in place of intensity values in building mean model and training the AAM. Given a new instance, Bayes classification provides the probability value of a pixel being a prostate (Fig. 2(e)). The centroid of the probability values (Fig. 2(f)) is utilized for automatic initialization of the AAM. Consequently, the probability map of the new instance is used for the propagation of the AAM in a multi-resolution framework (Fig. 2(g), 2(h) and 2(i)) to segment the prostate (Fig. 2(j)). Prior probability information obtained in the EM framework provides an improved prostate tissue model compared to raw intensities, in presence of intensity heterogeneities. Bayes classification with prior probabilities from EM and pixel position produces a more accurate representation of the prostate region. The probability values being close to the mean model, the difference with the mean model is considerably reduced. This in turn reduces fitting error producing an accurate prostate segmentation.

\section{EXPERIMENTAL RESULTS}

We have validated the accuracy and robustness of our approach with 24 TRUS images with a resolution of $354 \times 304$ pixels from 6 prostate datasets in a leave-one-patient-out evaluation strategy. We have used most of the popular prostate segmentation evaluation metrics like Dice Similarity Coefficient (DSC), 95\% Hausdorff Distance (HD), Mean Absolute Distance (MAD), Maximum Distance (MaxD), specificity, sensitivity, and accuracy to evaluate our method.

Table 1 shows the obtained results compared with the traditional AAM proposed by Cootes et al. [3] and our previous work [5]. It is observed that with respect to overlap accuracy and contour accuracy, our probabilistic information guided AAM performs better than traditional AAM [3] and the texture guided AAM [5]. This could be attributed to the fact that a probabilistic representation of the prostate region in TRUS images improves segmentation accuracy compared to the use of raw intensities [3] or texture [5]. We achieve a statistically significant improvement in $t$-test for DSC with $p=0.0027$ compared to traditional AAM [3] and $p=0.0009$ compared to our previous work [5]. Moreover, our proposal has a statistically significant improvement in $t$-test for $\mathrm{HD}$ and 
Table 2. Prostate Segmentation Evaluation Metrics Comparison for TRUS and MR Images

\begin{tabular}{|c|c|c|c|c|}
\hline Reference & Year & Area Overlap Accuracy & Contour Accuracy & Datasets \\
\hline Betrouni [6] & 2004 & Average Area overlap 93 $\pm 0.9 \%$ & Average distance $3.77 \pm 1.3$ pixels & 10 images \\
Shen [7] & 2003 & Average Area overlap error 3.98 $\pm 0.97 \%$ & Average distance $3.2 \pm 0.87$ pixels & 8 images \\
Ladak [8] & 2000 & Average Area accuracy 90.1 $\pm 3.2 \%$ & Average MAD $4.4 \pm 1.8$ pixels & 117 images \\
Cosio [9] & 2008 & - & Average MAD $1.65 \pm 0.67 \mathrm{~mm}$ & $22 \mathrm{images}$ \\
\hline Our Method & & Average DSC 0.96 \pm 0.01 & Average MAD $0.80 \pm 0.24 \mathrm{~mm} / 2.86 \pm 0.88$ pixels & 24 images $/ 6$ datasets \\
\hline
\end{tabular}

MAD with $p<0.0001$ compared to [3] and [5]. Note that a high DSC value and a low contour error metrics of $\mathrm{HD}$ and $\mathrm{MAD}$ are all equally important in determining the segmentation accuracy of an algorithm. In this context, we can claim that segmentation accuracy of our method is better compared to [3] and [5]. Our method is implemented in Matlab 7 on an Intel Core 2 Duo T5250, 1.5 Ghz processor and 2 GB RAM. The mean segmentation time is $5.95 \pm 0.05$ seconds.

The robustness of the proposed method against low SNR, intensity heterogeneities, speckle noise and microcalcification is illustrated in Fig. 3. On automatic initialization, our AAM successfully avoids the artifact and segments the prostate (black contour) with an accuracy of $98 \%$ (Fig. $3(\mathrm{c})$ ). To provide qualitative results of our method we present a subset of results obtained in Fig. 3(c), 3(d), 3(e), and 3(f). A quantitative comparison of different prostate segmentation methodologies is difficult in absence of a public dataset and standardized evaluation metrics. Nevertheless, to have an overall qualitative estimate of the functioning of our method we have compared our method with some of the works in the literature in Table 2 (a ' - ' in the table means information not available). Analyzing the results we observe that our mean DSC value is comparable to area overlap accuracy values of Betrouni et al. [6] and Ladak et al. [8] and very close to the area overlap error of Shen et al. [7]. However, it is to be noted that we have used more images compared to Shen et al. Our MAD value is comparable to [6], [7], [8] and to [9]. From these observations we may conclude that qualitatively our method performs well in overlap and contour accuracy measures. However, unlike $[6,7,8,9]$ the strength of our method lies in the probabilistic approach to the problem.

\section{CONCLUSION AND FUTURE WORKS}

A novel approach of AAM propagation from probabilistic texture information estimated in a Bayesian framework with the goal of segmenting the prostate in 2D TRUS images has been proposed. Our approach is accurate, computationally efficient and more robust in segmenting TRUS images compared to traditional AAM [3] and our previous work [5]. While the proposed method is validated with prostate mid gland images, effectiveness of the method against base and apical slices is yet to be validated with the extension of the model for 3D segmentation.

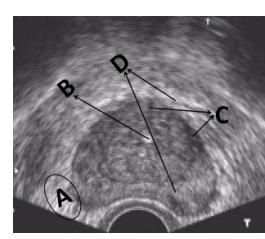

(a)

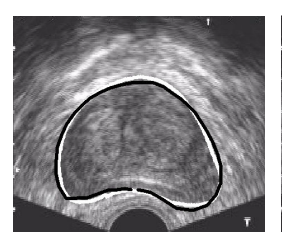

(d)

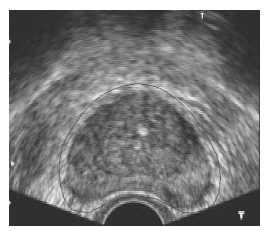

(b)

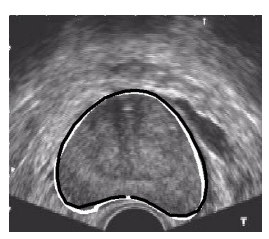

(e)

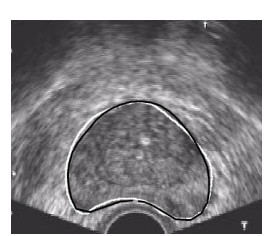

(c)

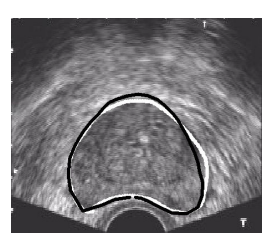

(f)
Fig. 3. (a) Artifacts in TRUS image of the prostate, $A=$ Low SNR, B=Micro Calcification, $\mathrm{C}=$ Intensity heterogeneity inside prostate, $\mathrm{D}=$ Speckle Noise. (b) Automatic initialization of the mean model, (c) Final segmentation result. (d), (e), and (f) shows some other examples of segmentation.

\section{REFERENCES}

[1] "Prostate Cancer Statistics - Key Facts," http://info.cancerresearchuk.org/cancerstats, 2010.

[2] A. J. Woodruff, T. M. Morgan et al., "Prostate volume as an independent predictor of prostate cancer and high-grade disease on prostate needle biopsy," Jrnl. of Clinical Oncology, vol. 26, pp. 5165, 2008.

[3] T.F. Cootes, G.J. Edwards et al., "Active Appearance Models," LNCS Springer, vol. 1407, pp. 484-498, 1998.

[4] R. O. Duda, P. E. Hart, and D. G. Stork, Pattern Classification, WileyInterscience, second edition, 2000.

[5] S. Ghose, A. Oliver et al., "Texture Guided Active Appearance Model Propagation for Prostate Segmentation," LNCS Springer, vol. 6367, pp. 111-120, 2010.

[6] N. Betrouni, M. Vermandel et al., "Segmentation of Abdominal Ultrasound Images of the Prostate Using A priori Information and an Adapted Noise Filter," Comp. Med. Imag. and Graphics, vol. 29, pp. 43-51, 2005.

[7] D. Shen, Y. Zhan et al., "Segmentation of Prostate Boundaries from Ultrasound Images Using Statistical Shape Model," IEEE Trans. on Med. Imag., vol. 22, pp. 539-551, 2003.

[8] H. M. Ladak, F. Mao et al., "Prostate Segmentation from 2D Ultrasound Images," Proc. IEEE EMBS, vol. 4, pp. 3188-3191, 2000.

[9] F. A. Cosío, "Automatic Initialization of an Active Shape Model of the Prostate," Med. Imag. Analysis, vol. 12, pp. 469-483, 2008. 